\title{
Optimizing Multiplexed Detections of Diabetes Antibodies via Quantitative Microfluidic
}

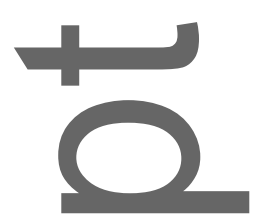

\section{Droplet Array}

Bioengineering Program, Department of Mechanical Engineering

Kai Duan, Gargi Ghosh, Joe Fujiou Lo*

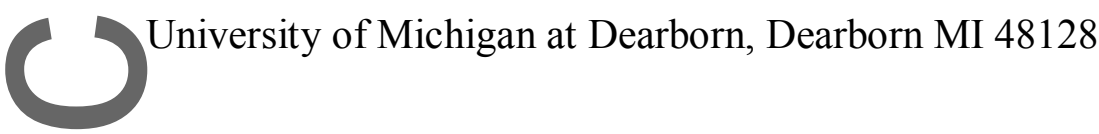

$\mathcal{\infty}$

*jfjlo@umich.edu

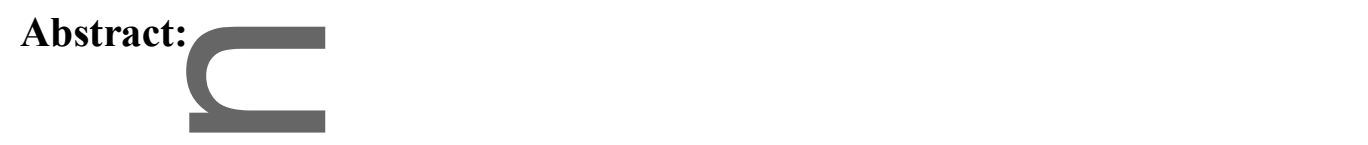

Sensitive, single volume detections of multiple diabetes antibodies can provide immunoprofiling and early screening of at-risk patients. To advance the state of the art suspension assays for diabetes antibodies, porous hydrogel droplets were leveraged in microfluidic serpentine arrays to enhance reagent transport. This spatially multiplexed assay was applied to the detection of antibodies against insulin, glutamic acid decarboxylase, and insulinoma associated protein 2. Optimization of assay protocol resulted in a shortened assay time of 2 hours, with better than $20 \mathrm{pg} / \mathrm{mL}$ detection limits across all three antibodies. Specificity and cross-reactivity tests showed negligible background, non-specific antibodyantigen, and non-specific antibody-antibody bindings. Multiplexed detections were able to measure within $15 \%$ of target concentrations from low to high ranges. The technique enabled quantifications of as little as 8000 molecules in each $500-\mu \mathrm{m}$ droplet in a single volume,

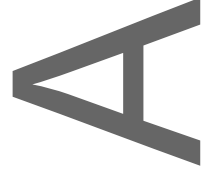

This is the author manuscript accepted for publication and has undergone full peer review but has not been through the copyediting, typesetting, pagination and proofreading process, which may lead to differences between this version and the Version of Record. Please cite this article as doi: 10.1002/smll.201702323.

This article is protected by copyright. All rights reserved. 
multiplexed assay format, a breakthrough necessary for the adoption of diabetes panels for clinical screening and monitoring in the future.

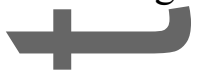

Keywords: Diabetes, Antibody, Microfluidic, Droplet, Multiplexing

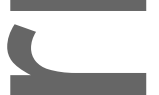

\section{Introduction:}

The role and function of the rising levels of autoantibodies in Type I diabetes (T1D) is of great interest to the disease pathogenesis. Detection of diabetes autoantibodies enables clinical diagnosis of $\mathrm{T} 1 \mathrm{D},{ }^{[1-10]}$ and sensitive detections have the potential to provide predictive and screening values. ${ }^{[10-13]}$ Autoantibodies against insulin, glutamic acid decarboxylase (GAD), and insulinoma associated protein-2 (IA -2) are well-established in T1D panels, and thus are targeted in multiplexed detections. ${ }^{[14,15]}$ The presence of IA-2 antibodies in clinical T1D ranges from 54-75\%. ${ }^{[16]}$ Additionally, $66 \%$ of newly diagnosed patients test positive for insulin autoantibody ${ }^{[17]}$. Testing for a panel of aforementioned antibodies using sensitive detections can identify better than $85 \%$ of disease presentation or future T1D development with 98\% specificity. ${ }^{[18]}$ Moreover, T1D accounts for more than $80 \%$ of diabetes in young children. ${ }^{[19]}$ Unsettlingly, development of T1D in children is especially acute, with afflicted children showing severe symptoms, very high blood glucose, marked glycosuria, and ketonuria. ${ }^{[20,21]}$ Incidentally, the rise of autoantibodies manifest very early, with transients observable before one year of age, Figure 1A, providing a strong marker for clinical diagnosis and screening for T1D. ${ }^{[22]}$ For these young patients, earlier diagnosis or even predictive screening may mean critical disease management before manifestation of life-threatening symptoms. Current understanding of these diabetes autoantibodies points to a potential neoantigen immunogenicity, ${ }^{[23-26]}$ where stress-modified protein synthesis leads to new epitopes on autoantigens that enhance their bindings to This article is protected by copyright. All rights reserved. 
antibodies $^{[27-31]}$ or elicit cell-mediated immune responses. ${ }^{[32-36]}$ Thus, sensitive detection of insulin, GAD, and IA-2 autoantibodies can serve as a powerful diabetes panel to monitor J

T1D disease progression, as well as investigate the mechanisms of beta cell autoimmune dysfunctions

The standard detection methods for diabetes autoantibodies include radioimmunoassays (RIAs) and enzyme-link immunosorbent assays (ELISAs). Serum RIA and ELISA protocols uses semi-quantitative titrations with varying sensitivities, which may not be useful for early or new-onset diabetics. ${ }^{[37]}$ In practice, RIA is time consuming and labor intensive, while ELISA requires multiple sample preparations to provide multiplexed detection of autoantibodies. ${ }^{[1,5]}$ Moreover, there is a need for a uniform method of quantitative detections of all three antibodies in a single volume. For example, serum autoantibody levels can be measured in dilution titers (e.g. Juvenile Diabetes Foundation units), RIA percentage, or in enzymatic $U / m L$, with various cut off definitions for positive detections. ${ }^{[1,5,15,38]}$ As a comparison, normal serum levels of all three autoantibodies are nominally regarded as 0.02 $\mathrm{nM}$ (e.g. $2.4 \mathrm{ng} / \mathrm{mL}$ for insulin $\mathrm{IgG}$ ) or lower by the World Health Organization and Mayo Clinic Interpretive Handbook ${ }^{[39]}$. Nevertheless, the cut-offs established by the various methods represent the diagnostic levels of T1D, whereas early screening would call for greater sensitivity to detect earlier transients.

Towards improving diabetes autoantibody detections, advances have been made via multiplexed microarrays ${ }^{[15]}$ and electrochemiluminescence (ECL) assays. ${ }^{[14,40-41]}$ Diabetes microarrays enabled single serum preparation for a multiplexed antibody detection panel, while ECL assays pushed the quantitative autoantibody detections below $10^{-11}$ mole/L $(\mathrm{ng} / \mathrm{mL})$ range, respectively. However, these separate improvements to autoantibody 
detections have not yet offered a combined sensitivity and multiplexing in a single volume assay.

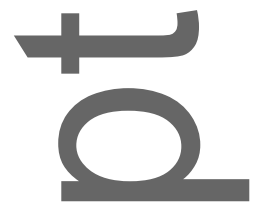

A)

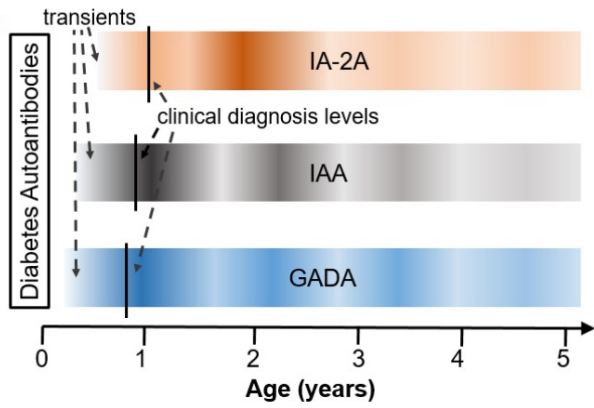

C)

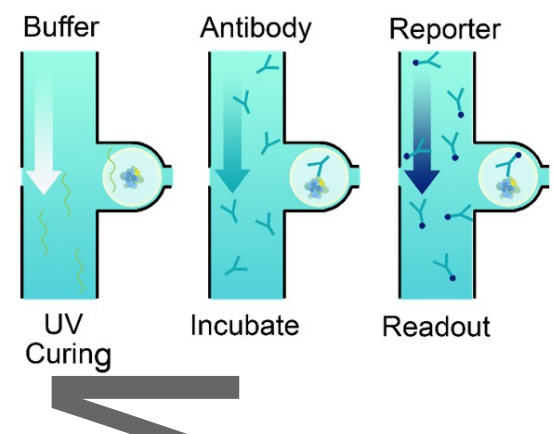

B)

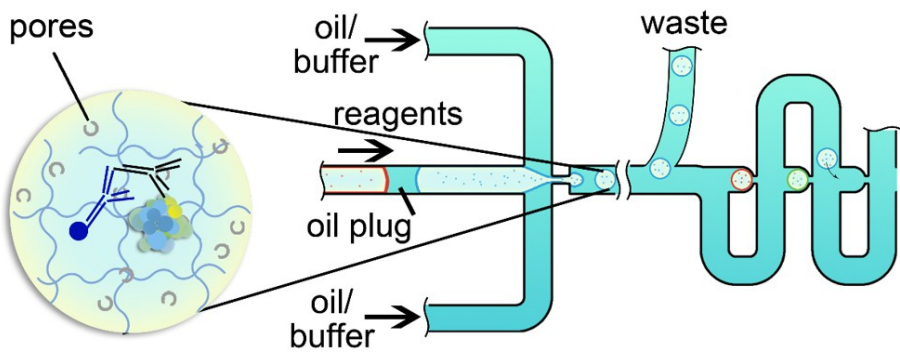

D)

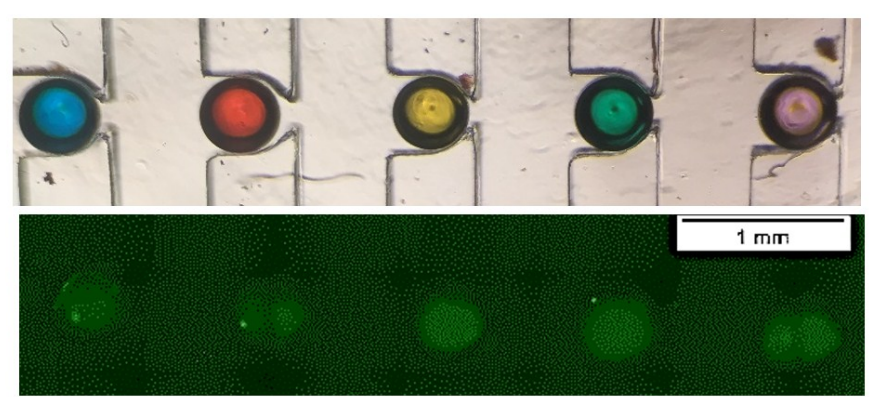

Figure 1. Quantitative microfluidic droplet array for diabetes detection panel. A) The rise and change of diabetes autoantibodies vary in individuals, and can be observed as early as 1 year of age, demonstrating the need for sensitive detection of multiple autoantibodies. B) We engineered a microfluidic array that generated individual porous droplets with detection chemistries optimized for the insulin, GAD, and IA-2 antibody detections. These "smart microgels" were then spatially arrayed in designated traps within the serpentine channels. This spatial multiplexing was achieved by flow switching of unwanted droplets to a waste channel, and redirecting droplets back towards the traps when the correct detection chemistry is generated. C) The serpentine microchannels allowed trapped droplets to be perfused by assay reagents. Buffer perfusion after UV curing allowed the porogen molecules (yellow stripes) to be washed away, creating a porous hydrogel droplet. Target antibodies (blue) and reporter antibodies (green) were then subsequently introduced into the microchannels, 
completing the immunoassay protocol. D) The result of spatial multiplexing was an array of droplets targeting individual diabetes antibodies, illustrated on top by the generation of multicolored droplets using food dyes. In the actual assay on bottom, only a single reporter wavelength was required, providing fluorescence intensities that varied at each spatial position per its respective antibody detection.

We addressed these needs for the diabetes detection panel by creating an enhanced suspension assays multiplexed in microfluidic channels. Standard suspensions assays based on polystyrene beads, hydrogels, multi-colored Luminex beads, Ilumina's VeraCode particles, and noyel plasmonic mulptilexed substrates share advantages of flexible probe chemistry, inert surfaces, radial diffusion, smaller sample size, and lower costs. ${ }^{[15,42-47]}$ Our group has further advanced the suspension assay technology by creating porous polyethylene glycol (PEG) hydrogel—“smart microgels"- to enhance the diffusion of biomolecules within the droplets, an improvement to the microscaled substrate that defines suspension assays. ${ }^{[48-}$ ${ }^{50]}$ In that previous work, we have demonstrated that the porosity generated by $20 \mathrm{kDa}$ PEG optimized the diffusion of $250 \mathrm{kDa}$ FITC-dextran into the hydrogel. This is larger than the 65 $\mathrm{kDa}$ antibodies used in this multiplexed assay, ensuring analyte access through the droplet volume. Furthermore, these microgels are arranged in a microfluidic serpentine array for multiplexing. ${ }^{[51]}$ The microfluidic flow pushes the soluble reagents closer to the substrate surface, which is otherwise shielded by the boundary layers in bulk flows. These porosity and microfluidic advantages enhance the reagent transport and turnover throughout the whole droplet volume, enabling $\mathrm{pg} / \mathrm{mL}$ protein detections with shortened assay time of less than 2 hours in previous works. ${ }^{[50,51]}$ Here, we leveraged our smart microgels further for multiplexed detection of diabetes antibodies, aided by the microfluidic enhancement of analyte transport (Figure S1). The multiplexed diabetes panel was optimized with recombinant proteins and antibodies to push the performance envelop of detections, towards 
serum measurements in the future. The result is a quantitative, faster, and single sample multiplexed detection panel for insulin, GAD, and IA2 antibodies. Finally, the platform can be easily adapted for future antibodies such as zinc transporter $8 .{ }^{[52-54]}$ Potential clinical applications of this detection panel may enable detailed immune profiling in T1D, and pave the way for a robust tool for early screening.

\section{Results and Discussion}

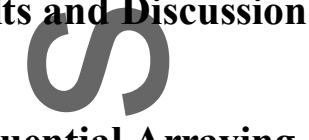

\subsection{Sequential Arraying of Enhanced Droplets for Multiplexed Detection}

Smart microgel optimizations were leveraged for the detection of diabetes antibodies by immobilizing capture antigens instead of capture antibodies. Detection chemistry, including diacrylated antigens in polyethylene diacrylate hydrogels and PEG porogen, were loaded into a microfluidic cross-junction, Figure 1B. Flow from this aqueous phase was then pinched by mineral oil at the junction, creating an instability that ejects $500 \mu \mathrm{m}$ droplets at a predictable frequency. ${ }^{[50]}$ Droplets then flowed down the serpentine channels and occupied individual trap sites one after another. However, in order to generate multiplexed detections for insulin, GAD, and A-2 antibodies in a single channel, droplets with different capture molecules were generated and sequentially trapped. This was achieved by directing the unwanted droplets towards a waste channel, then switching the flow towards the serpentine traps once desired droplets were generated, Figure 1B. Upon trapping, the droplets were UV cured to immobilize the capture antigens and solidify the hydrogel for washing in TBST (Trisbuffered Saline and Tween 20) to remove porogens, Figure 1C. With porosity-enhanced mass transport, unreacted porogen was thoroughly removed after 4.5 hours of microfluidic perfusion, compared to the 24 hours of incubation needed for bulk preparations. ${ }^{[50]}$ The resultant array, Figure 1D, represented a spatially multiplexed diabetes detection panel, 
without issues associated with spectral overlapping or computational imaging algorithms for shape-coded techniques. After formation of the multiplexed smart microgels, the array was then ready to be assayed by flowing target antibodies, reporter antibodies, and washing steps, optimized in the following section.

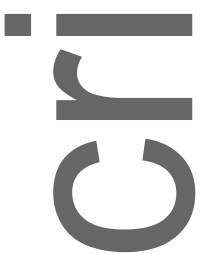

\subsection{Optimization of Reporter Incubation Time and Dilution Factor}

The detections of all three antibodies shared a common reporter, FITC-labeled IgG, whose incubation time and dilution factor were optimized using the insulin antibody detection. The fluorescence readout from the detection was quantified by the maximum droplet intensity divided by the background intensity, as illustrated by the line scan in Figure 2A. Using this quantification, reporter readout plateaued after 40 minutes of incubation, Figure 2 B. Furthermore, the reporter dilution showed lowered background and useful signal up to 1024 $\mathrm{X}$ dilution, which dramatically reduced the usage of reporter reagents, Figure $2 \mathrm{C}$. Based on these results, all subsequent experiments were carried out with 40 minutes of reporter incubation at $1024 \mathrm{X}$ dilution. We anticipate the lower background from reporter dilution may help reduce non-specific signals when moving to serum-based detections in the future.
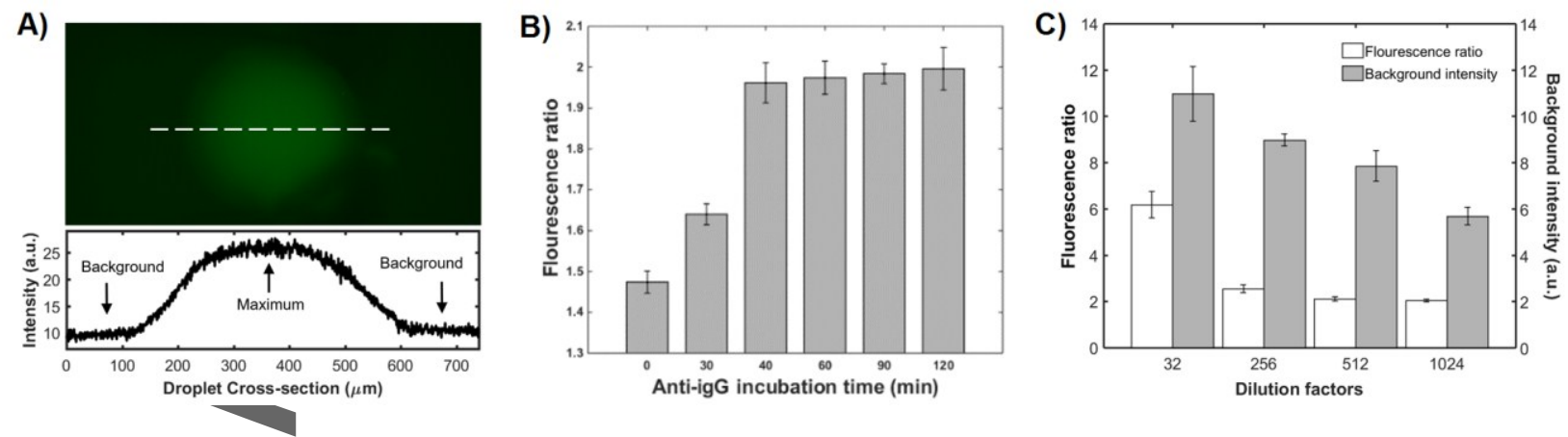

Figure 2. Reporter optimization. A) All fluorescence measurements were quantified by the ratio of maximum center intensity normalized by the background intensity, as seen by the line 
scan across a typical droplet. B) Fluorescence ratio of the reporter plateaued after 40 minutes of reporter incubation. C) While fluorescence ratio flattened after 256 times reporter dilution, the noise continued to drop. We selected the reporter dilution of 1024 times to reduce reagent consumption and minimize the background intensity, which would provide smaller standard deviations for more sensitive detections.

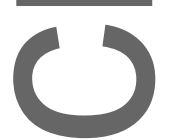

\subsection{Optimization of Capture Antigens Concentration in PEGDA 02}

After reporter optimization, the capture antigen concentration corresponding to each target antibody was optimized. All antigen optimizations were incubated with $1000 \mathrm{pg} / \mathrm{mL}$ target antibodies. Figure 3A illustrates the insulin capture antigen optimization, where the fluorescence ratio plateaued above a concentration of $200 \mathrm{pg} / \mathrm{mL}$. GAD antigen optimization is shown in Figure 3B, where the fluorescence ratio showed a plateau above $500 \mathrm{pg} / \mathrm{mL}$. Similarly, Figure 3C shows that IA-2 concentration in PEGDA plateaus above $500 \mathrm{pg} / \mathrm{mL}$. Optimization of antigen immobilization illustrated the differences of the binding epitopes between each antigen-antibody pair. ${ }^{[23-36]}$ The subsequent experiments were run at their respective optimized antigen concentrations.

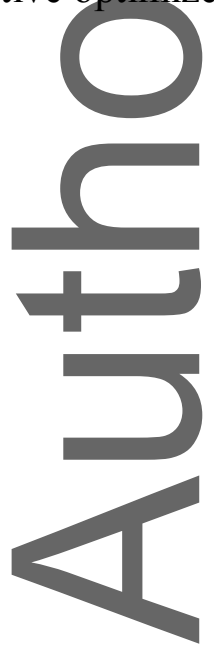



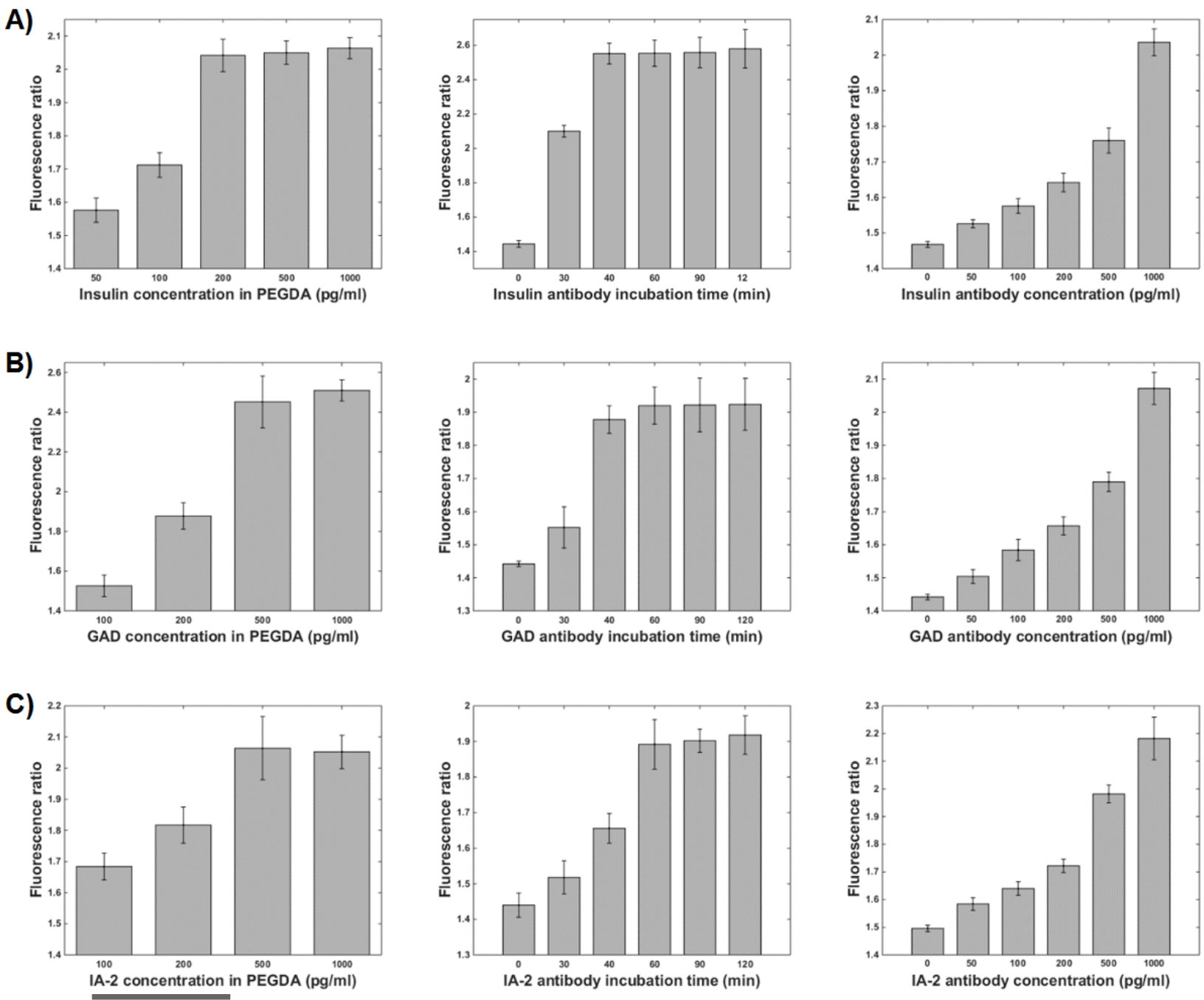

Figure 3. (Diabetes panel optimization. A) Insulin antibody detection was optimized with $200 \mathrm{pg} / \mathrm{mL}$ capture antigen immobilization, 40 minutes of analyte incubation, and a detectivity curve with a detection limit of $19.6 \mathrm{pg} / \mathrm{mL}$. B) GAD antibody detection was optimized with a $500 \mathrm{pg} / \mathrm{mL}$ capture antigen immobilization, 40 minutes of analyte incubation, and a detectivity curve with a detection limit of $18.7 \mathrm{pg} / \mathrm{mL}$. C) IA-2 antibody detection was optimized with a $500 \mathrm{pg} / \mathrm{mL}$ capture antigen immoblization, 60 minutes of analyte incubation, and a detectivity curve with a detection limit of $12.7 \mathrm{pg} / \mathrm{mL}$. All capture antigen and analyte incubation optimizations were completed in triplicates. All detectivity curves were completed with 5 replicate runs. Error bars denote standard deviations.

\subsection{Optimization of Target Antibodies Incubation Time}

This article is protected by copyright. All rights reserved. 
Next, we considered the kinetics of antibody-antigen binding within the smart microgels by optimizing the target antibody incubation times. Smart microgels immobilized with aforementioned antigen concentrations were incubated for various durations with their respective-antibodies. The fluorescence ratio for insulin detection improved up to 40 minutes of target antibody incubation and plateaued thereafter, Figure 3A. The same was seen for GAD antibody with a plateau after 40 minutes, Figure 3B. IA-2 and its antibody showed a different kineties, as optimal incubation did not occur until after 60 minutes, Figure 3C. We should also note that IA-2 antibody is sensitive to buffer conditions, where glycine additives would increase non-specific binding to the PEG hydrogel. Based on these optimizations, the longest incubation time was 60 minutes for the IA-2 antibody, which we adopted for all subsequent singleplexed and multiplexed detections.

\subsection{Characterizing the Limits of Antibody Detections}

The assay protocol described above was optimized at $1000 \mathrm{pg} / \mathrm{mL}$ for respective diabetes antibodies. We subsequently tested the detectivity below this concentration to characterize the detection limits for each antibody. Concentrations of 50,100, 200, 500, and $1000 \mathrm{pg} / \mathrm{mL}$ were assayed in five runs for each antibody, with error bars denoting standard deviations between each assay run at the particular concentration, Figure 3. The detection limit was defined to be the concentration at which the signal rises three times the standard deviations of the zeroth concentration. Using this definition, the insulin antibody detection limit was found to be $19.6 \mathrm{pg} / \mathrm{mL}$, Figure 3A. The GAD antibody detection limit was found to be 18.7 $\mathrm{pg} / \mathrm{mL}$, Figure 3B. And the IA-2 antibody detection limit was found to be $12.7 \mathrm{pg} / \mathrm{mL}$, Figure $3 \mathrm{C}$. Since the detection limit is a function of the background fluorescence and slope of the detectivity curve, minimization of reporter antibody contributed to these enhanced detection limits, Figure 2C. 


\subsection{Characterization of Assay Specificity}

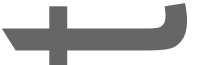

To investigate whether other protein components in serum could potentially affect the fluorescence ratio, antigen immobilized microgel droplets were incubated with phosphate buffered saline (PBS), 0.1\% bovine serum albumin (BSA) and their respective antibodies at $1000 \mathrm{pg} / \mathrm{mL}$, Figure 4. Antibody detections yielded fluorescence ratios higher than 2, whereas PBS and BSA yielded background levels of 1.5 or lower. More importantly, errors bars showed significant differences between specific and background detections with $\mathrm{p}<0.0001$. One reason for the low background without blocking is the bioinert nature of PEG hydrogel against non-specific antibody binding. The result indicated that proteins other than targeted diabetes antibodies had minimum contributions towards the fluorescent signal, and also demonstrated that antigen functionalization without additional blocking is sufficient for smart microgel specificity.

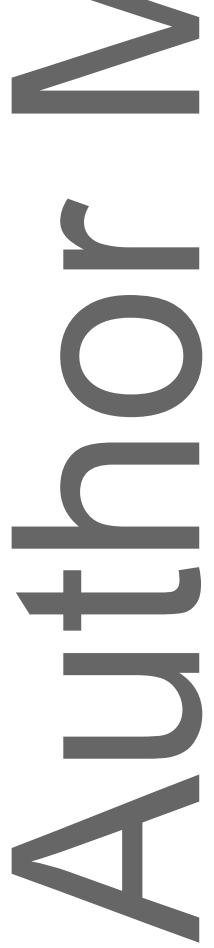




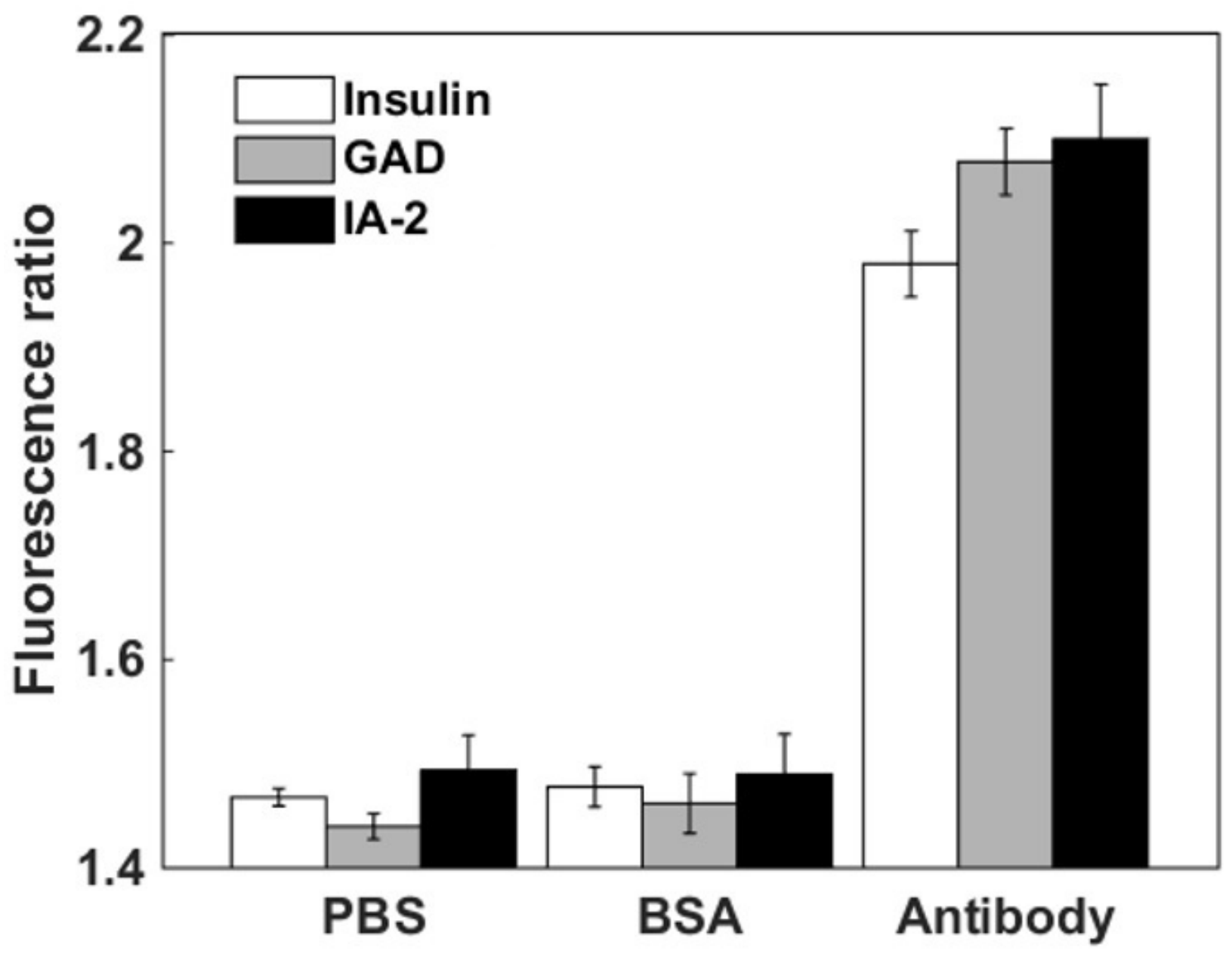

Figure 4. Assay specificity. Non-specific binding to BSA resulted in fluorescence ratios on par with PBS background. Specific antibody detections resulted in a ratio around 2 versus 1.5 of background. Moreover, significant differences between detections and background yielded a $\mathrm{p}<0.0001$

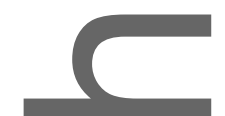

\subsection{Characterization of Singleplex and Duplex Cross-reactivity}

One major diffieulty in assaying multiple antibodies within one sample assay volume is the cross-reaetivity among the target antibodies and non-target proteins. First, microgel droplets of all target antigens were incubated with each target antibody one at a time at $1000 \mathrm{pg} / \mathrm{mL}$ to evaluate antigen-antibody cross-reactivity, Figure 5. For reference, background fluorescence ratios for each antigen (zero antibody concentrations) were typically below 1.5 , as seen from 
their detectivity curves. In comparison, the on-target antigen-antibody detections, e.g. insulin to insulin antibody, yielded a ratio close to 2, significantly higher than the background ( $p<0.0001$, complete comparisons in Table S1). After analysis of singleplex cross-reactivity, combinations of two antibodies each at $1000 \mathrm{pg} / \mathrm{mL}$ were assayed, Figure 6. As singleplex cross-reactivity showed negligible nonspecific bindings between antibody and capture antigens, this duplex assay tested the possibility of unintended antibody to antibody interactions. Again, the on-target pairs yielded fluorescence ratios close to 2, while missmatched backgrounds remained below 1.5. For example, the GAD to insulin $+\mathrm{GAD}$ antibodies fluorescence ratio was significantly higher than that of the IA-2 mismatched with insulin + GAD antibodies $(p<0.0001,2 D$ plots and complete statistics in Figure S2 and Table S1).

A)

\section{I}

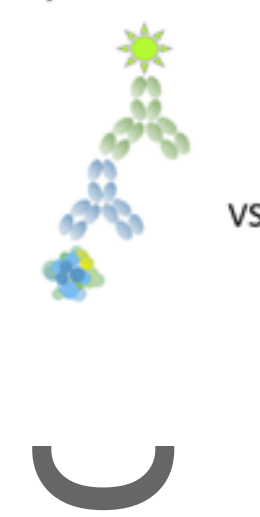

B)

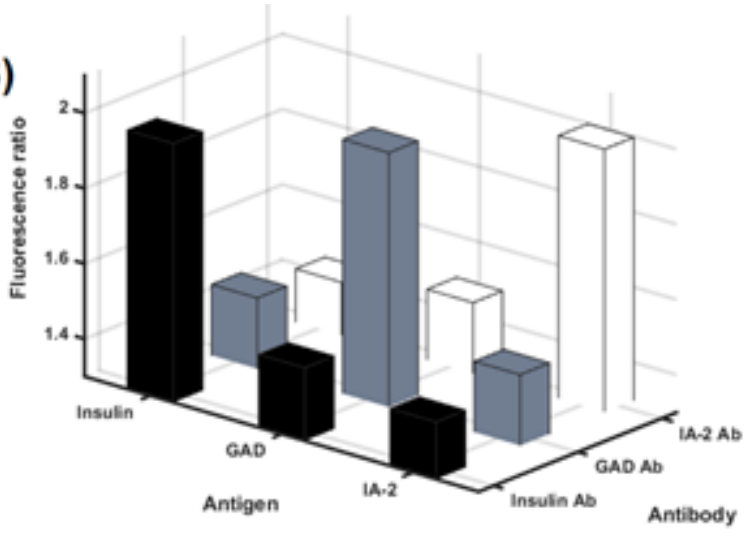

Figure 5. Singleplex cross-reactivity. A) Cross-reactivity between target (blue) and nontarget (orange) antibodies one at a time tested non-specific antibody-antigen interactions. B) This test showed that non-specific antibody-antigen reactions yielded fluorescence ratios similar to that of background at $\sim 1.5$.

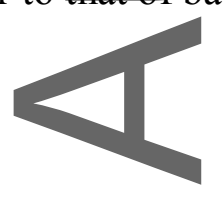


A)

vs.

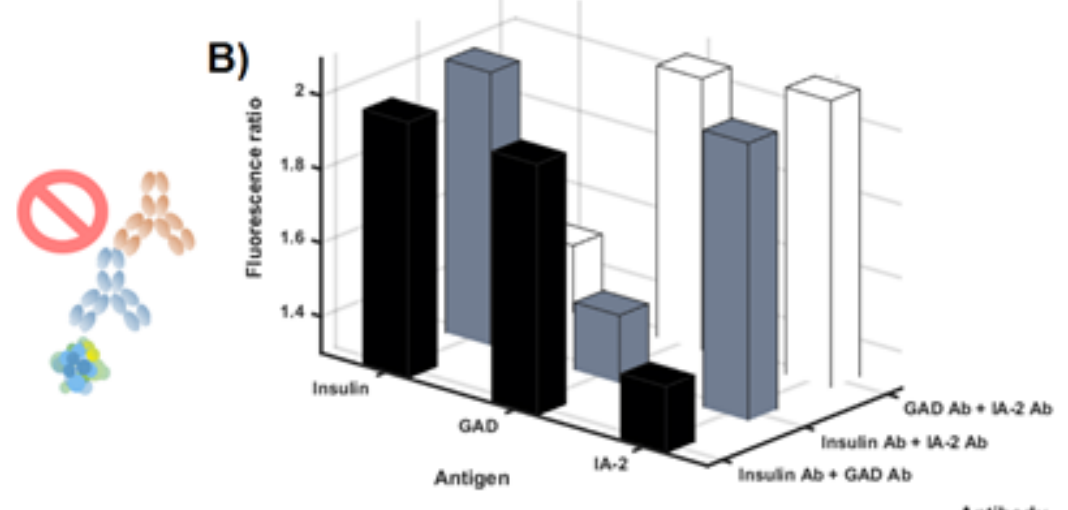

Figure 6. Duplex cross-reactivity. A) Cross-reactivity between combinations of antibodies pairs tested non-specific antibody-antibody interactions. B) This test showed that nonspecific antibody-antibody reactions did not affect the on-target antibody-antigen detections.

\subsection{Muliplexed Detection and Quantitative Protein Recovery}

After confirming negligible cross-reactivities at elevated antibody concentrations, we demonstrated multiplexed detections of insulin, GAD, and IA-2 antibody concentrations and their quantifications simultaneously. Three concentrations of 50, 200, and $500 \mathrm{pg} / \mathrm{mL}$ were assayed for each target antibody in multiplexed droplets. Triplicates were run for each condition. However, since the detections were multiplexed, the entire assay took a total of just 9 runs. The measured concentrations were within $15 \%$ of the designated values, Table 1 .

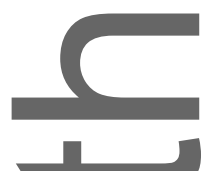

Table 1. Multiplexed protein detections with target concentrations of 50, 200, and 500 $\mathrm{pg} / \mathrm{mL}$ for all three antibodies simultaneously.

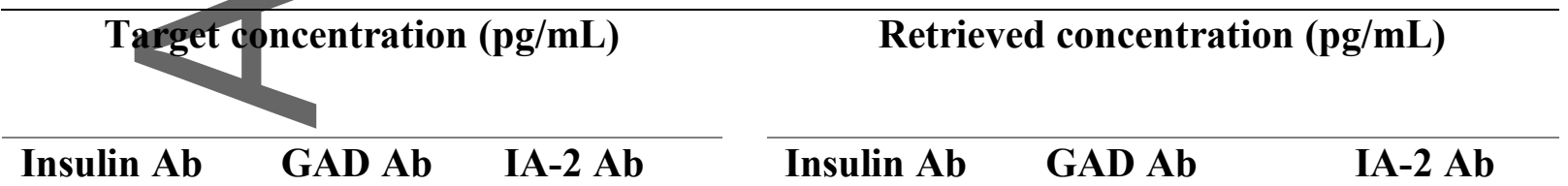




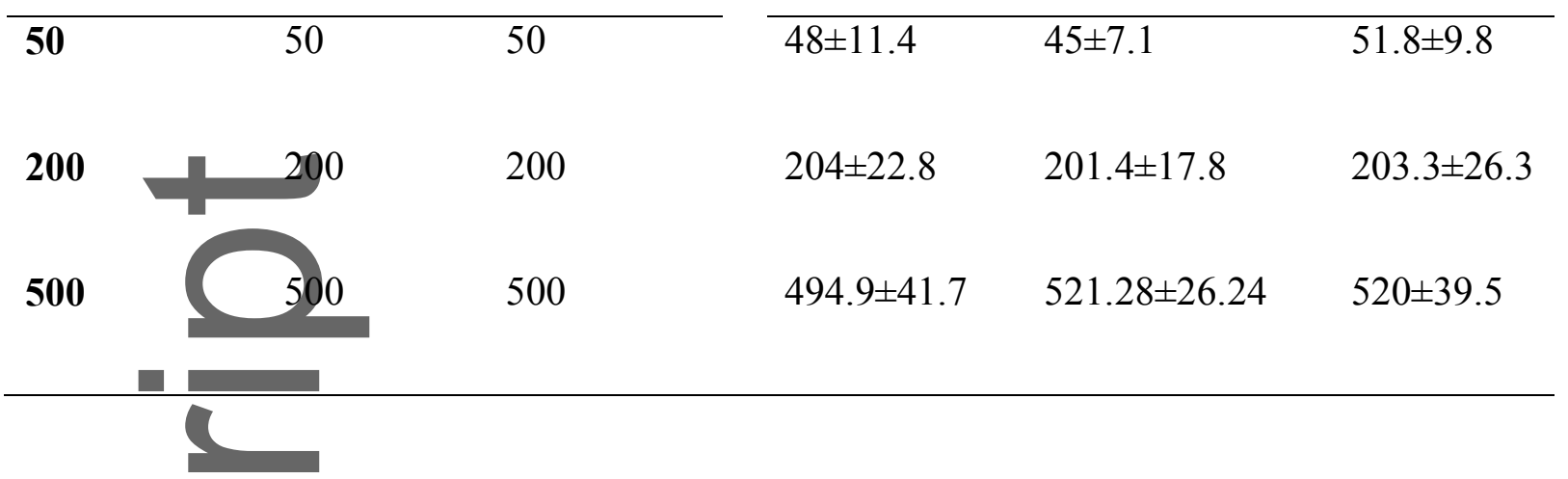

\section{Discussion}

The diabetes detection panel presented here achieved detection limits better than $20 \mathrm{pg} / \mathrm{mL}$ for all three target antibodies optimized. For reference, the recombinant antibodies used in this detection panel weighed less than $65 \mathrm{kDa}$, which means around just 8 to 30 thousand molecules were detected in each $500 \mu \mathrm{m}$ droplet. This level of detection is orders of magnitude below serum levels of insulin, GAD, and IA-2 autoantibodies in non-diabetic patients, nominally at $2.4 \mathrm{ng} / \mathrm{mL}$. However, that threshold for T1D diagnosis does not account for the low level transients before the rise of antibody levels in serum, which can be present in patients as young as one year of age. ${ }^{[22]}$ With a sensitive, multiplexed detection panel, at-risk patients and siblings or family members of diabetics can receive early screening and frequent monitoring for the development of diabetes, before any hyperglycemia has manifested.

One major benefit of the smart microgel antibody detection is its inert hydrogel chemistry, which eliminates the need for blocking and reduces the assembly time for each assay. Next, the spatial multiplexing eliminates the possibility of spectral overlapping or complex shape coded imaging. Furthermore, the length for target incubation, reporter incubation, and two buffer washing times totaled 2 hours - an accelerated protocol that is difficult to achieve by current antibody or RIA assays. Since the protein reagents are suspended in hydrogel substrates, their native conformation and binding kinetics can be preserved. ${ }^{[55-57]}$ Moreover, 
enhanced hydrogel based substrates may demonstrate better storage capabilities ${ }^{[58-60]}$, enabling pre-generated, pre-packaged diabetes detection panels for wider distribution and assay adaptation. Additionally, newly identified neoantigens ${ }^{[23-36]}$ and $\mathrm{ZnT} 8^{[52-54]}$ can be rapidly added in a future multiplexed version of the diabetes panel, to enable clinical investigations of their predicative and screening values ${ }^{[4-7]}$, plus potential disease mechanisms. Lastly, available hydrogel functionalities can also enable immobilization of short peptides, nucleotides, aptamer, reverse transcription amplification, or cell encapsulates to create novel detection schemes or beta cell stimulations ${ }^{[61-66]}$, all of which can benefit from the enhanced hydrogel kinetics and multiplexing presented in this work. Coupled with the microfluidic serpentine's ability to manipulate soluble concentrations, the smart microgel platform provides a highly useful in vitro tool to study diabetes mechanisms.

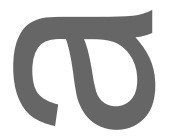

\section{Conclusion}

We have achieved a multiplexed detection panel for diabetes antibodies targeting insulin, GAD, and IA-2. The assay can be prepared in 4.5 hours prior to running the assay, with a total assay time of just 2 hours for all three parameters in a single sample. For all target antibodies, a detection limit better than $20 \mathrm{pg} / \mathrm{mL}$ was achieved, representing the detection of only 8 to $30 \mathrm{k}$ molecules in each microgel droplet. Singleplex and multiplex detections showed negligible cross-reactivity. Multiplexed detections were within $15 \%$ of the target concentrations. The serpentine microfluidic achieved a spatial multiplexing of the microgels that avoided isstes associated with spectral or imaging based techniques. The resultant detection panel has the potential to improve diabetes detection as well as investigate immunogenic mechanisms in diabetes.

\section{Experimental Section}


Reagents preparations: Yeast host recombinant human insulin, recombinant protein GAD 2, anti-GAD 65 (mouse anti-human), and recombinant IA-2 proteins were procured from SigmaAldrich. Mouse anti-human anti-insulin, anti-IA2 antibodies (mouse anti-human), and goat anti-mouse FITC tabeled IgG were procured from Millipore. Poly(ethylene) glycol diacrylate $(\mathrm{PEGDA})(\mathrm{Mn} \sim 6000)$, poly(ethylene glycol) $(\mathrm{PEG})(\mathrm{Mn} \sim 20,000)$, photo-initiator (2hydroxy-4'-(2-hydroxyethoxy)-2 methylpropionphenone), phosphate buffer saline (PBS), and albumin from bovine serum (BSA) were procured from Sigma-Aldrich. Acrylate poly (ethylene) glycol succinimidyl carboxymethyl MW 5000 (ACRL-PEG-SCM-5000) was obtained from Laysan Bio Inc. (Arab, AL).

To immobilize the antigens within the microgel, the antigens were incubated with $1.25 \mathrm{mg}$ of ACRL-PEG-SCM-5000 in PBS at room temperature for 3 hours prior to use and discarded afterwards. The pre-polymer solution consisting of $100 \mu 1$ of $8 \%$ (w/v) of PEG6000DA, 2\% (w/v) photo initiator, $10 \%(\mathrm{w} / \mathrm{v})$ PEG $20 \mathrm{~K}$, and acrylated antigen was then loaded in the microfluidics for droplet generation.

Droplet generation: The pre-polymer solution was introduced at $1 \mu \mathrm{L} / \mathrm{min}$, and mineral oil was introduced at $15 \mu \mathrm{L} / \mathrm{min}$ to generate the droplets at the microfluidic cross-junction. When one of the generated droplets was flown in to the serpentine pockets, its trapping blocks the cross-serpentine flow and redirects the next droplet towards the following pocket sequentially. After trapping, droplets were exposed to ultraviolet light for crosslinking for 5 minutes (UVP CL-1000 UV Oven, $365 \mathrm{~nm}$ ). Then, BSA buffer was flown in at $30 \mu \mathrm{L} / \mathrm{min}$ to continuously wash the droplet for 30 minutes to prevent PDMS protein absorption. Lastly, PBS was flown in at $30 \mu \mathrm{L} / \mathrm{min}$ to continuously wash the droplets for 4 hours to remove the unreacted reagents and PEG porogens, thus creating the porous microgel. 
Assay protocol: For multiplexed detection, different pre-polymers containing different acrylated antigens were loaded in the microfluidic tubing (Tygon tubing 1/16" ID) separated by mineral oil plugs. The pre-polymers were then delivered into the microfluidics using a syringe pump to generate droplets. After generating and trapping droplets with the first prepolymer reagent, subsequent unwanted droplets were redirected to the waste channel. Then, the waste channel was closed to allow droplets with the second pre-polymer containing the next acrylated antigen to be flown in and trapped in the next serpentine trap. The process continues untin all three pre-polymers containing the three different capture antigens were trapped in designated spots. These smart microgel droplets were then UV-cured and washed as described above.

To run the-multiplexed assays, smart micorgels were incubated with appropriate antibodies at $20 \mu \mathrm{L} / \mathrm{min}$ flow rate for 60 minutes. Then, they were washed with TBST at $30 \mu \mathrm{L} / \mathrm{min}$ for 15 minutes and subsequently incubated with the reporter antibody at $30 \mu \mathrm{L} / \mathrm{min}$ for 40 minutes. Following this, droplets were washed again with TBST for 5 minutes and then imaged under the fluorescence microscopy.

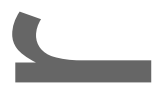

Fluorescence data and statistical analysis: After all the reactions between antigens, antibodies and reporter antibody were completed, the smart microgel droplets were imaged under fluorescence microscopy for readout. The ratio of maximum center droplet intensity over background intensity were calculated using ImageJ to provide a normalized intensity value. The noise of the data is then the standard deviations of the normalized values. All optimization and eross-reactivity were done in triplicates, while the detection curve was done with five repeated runs. To generate the statistics, detections were compared to background by individual-samples $t$-test. The p-values (double sided) were provided for interpretation, Table S1. 


\section{Acknowledgements}

This research was supported by the NIH 1R03EB023459 grant and the University of

Michigan at Dearborn Office of Research.

\section{Conflict of Interest}

The authors dectare no conflict of interest.

\section{References}

1. C. Törn, P. W. Mueller, M. Schlosser, E. Bonifacio, P. J. Bingley. Diabetes Antibody Standardization Program: evaluation of assays for autoantibodies to glutamic acid decarboxylase and islet antigen-2. Diabetologia. 2008; 51(5):846-52.

2. T. P. Staeva, L. Chatenoud, R. Insel, M. A. Atkinson. Recent lessons learned from prevention and recent-onset type 1 diabetes immunotherapy trials. Diabetes. 2013; $62(1): 9-17$.

3. J. M. Wenzlau, J. C. Hutton. Novel diabetes autoantibodies and prediction of type 1 diabetes. Current diabetes reports. 2013; 13(5):608-15.

4. C. J. Greenbaum, J. P. Palmer, B. Kuglin, H. Kolb. Insulin autoantibodies measured by radioimmunoassay methodology are more related to insulin-dependent diabetes mellitus than those measured by enzyme-linked immunosorbent assay: results of the Fourth International Workshop on the Standardization of Insulin Autoantibody Measurement. The Journat of Clinical Endocrinology \& Metabolism. 1992; 74(5):1040-4. 
5. M. Schlosser, P. W. Mueller, C. Törn, E. Bonifacio, P. J. Bingley. Diabetes Antibody Standardization Program: evaluation of assays for insulin autoantibodies. Diabetologia. $2010 ; 53(12): 2611-20$.

6. V. Lampasona, M. Schlosser, P. W. Mueller, A. J. Williams, J. M. Wenzlau, J. C. Hutton, P. Achenbach. Diabetes antibody standardization program: first proficiency evaluation of assays for autoantibodies to zinc transporter 8. Clinical chemistry. 2011; $57(12): 1693-702$

7. P. J. Bingley, E. Bonifacio, P. W. Mueller. Diabetes Antibody Standardization Program: first assay proficiency evaluation. Diabetes. 2003; 52(5):1128-36.

8. M. A. Atkinson, N. K. Maclaren, W. J. Riley, W. E. Winter, D. D. Fisk, R. P. Spillar. Are insulin autoantibodies markers for insulin-dependent diabetes mellitus? Diabetes. $1986 ; 35(8): 894-8$.

9. A. J.Williams, V. Lampasona, M. Schlosser, P. W. Mueller, D. L. Pittman, W. E. Winter, B. Akolkar, R. Wyatt, C. Brigatti, S. Krause, P. Achenbach. Detection of antibodies directed to the N-terminal region of GAD is dependent on assay format and contributes to differences in the specificity of GAD autoantibody assays for type 1 diabetes. Diabetes. 2015:db141693.

10. C. Andersson, M. Kolmodin, S. A. Ivarsson, A. Carlsson, G. Forsander, B. Lindblad, J. Ludvigsson, I. Kockum, C. Marcus, U. Samuelsson, E. Örtqvist. Islet cell antibodies (ICA) identify autoimmunity in children with new onset diabetes mellitus negative for other islet cell antibodies. Pediatric diabetes. 2014; 15(5):336-44.

11. J. M. LaGasse, M. S. Brantley, N. J. Leech, R. E. Rowe, S. Monks, J. P. Palmer, G. T. Nepom, D. K. McCulloch, W. A. Hagopian. Successful prospective prediction of type 1 
diabetes in schoolchildren through multiple defined autoantibodies. Diabetes Care. $2002 ; 25(3): 505-11$.

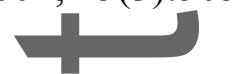

12. C. F. Verge, R. Gianani, E. Kawasaki, L. Yu, M. Pietropaolo, H. P. Chase, G. S. Eisenbarth, R. A. Jackson. Prediction of type I diabetes in first-degree relatives using a combination of insulin, GAD, and ICA512bdc/IA-2 autoantibodies. Diabetes. 1996; $45(7): 926-33$

13. A. G. Ziegler, S. Schmid, D. Huber, M. Hummel, E. Bonifacio. Early infant feeding and risk of developing type 1 diabetes-associated autoantibodies. Jama. 2003; 290(13):17218.

14. Z. Zhao, D. Miao, A. Michels, A. Steck, F. Dong, M. Rewers, L. Yu. A multiplex assay combining insulin, GAD, IA-2 and transglutaminase autoantibodies to facilitate screening for pre-type 1 diabetes and celiac disease. Journal of immunological methods. $2016 ; 430: 28-32$.

15. B. Zhang, R. B. Kumar, H. Dai, B. J. Feldman. A plasmonic chip for biomarker discovery and diagnosis of type 1 diabetes. Nature medicine. 2014; 20(8):948-53.

16. L. Herszenyi, Z. Tulassay. The role of autoantibodies in inflammatory bowel disease. Digestive Diseases. 2012; 30:201-207.

17. L. Yu, D. T. Robles, N. Abiru, P. Kaur, M. Rewers, K. Kelemen, G. S. Eisenbarth. Early expression of antiinsulin autoantibodies of humans and the NOD mouse: evidence for early determination of subsequent diabetes. Proceedings of the National Academy of Sciences. 2000; 97:1701-1706.

18. P. J. Bingley, E. Bonifacio, A. G. Ziegler, D. A. Schatz, M. A. Atkinson, G. S. Eisenbarth. Proposed guidelines on screening for risk of type 1 diabetes. Diabetes Care 
2001; 24(2): 398-398.

19. SEARCH for Diabetes in Youth Study Group. The burden of diabetes mellitus among US youth: prevalence estimates from the SEARCH for Diabetes in Youth Study. Pediatrics 2006; 118(4): 1510-1518.

20. C. C.Patterson, G. G. Dahlquist, E. Gyürüs, A. Green, G. Soltész; EURODIAB Study Group. Incidence trends for childhood type 1 diabetes in Europe during 1989-2003 and predicted new cases 2005-20: a multicentre prospective registration study. The Lancet 2009; 373(9680): 2027-2033.

21. K. G. M. M. Alberti, P.Z. Zimmet. Definition, diagnosis and classification of diabetes mellitus and its complications. Part 1: diagnosis and classification of diabetes mellitus. Provisional report of a WHO consultation. Diabetic medicine 1998; 15(7): 539-553.

22. E. Bonifacio. Predicting type 1 diabetes using biomarkers. Diabetes Care. 2015; 38(6):989-96.

23. M J. Kracht, A. Zaldumbide, B. O. Roep. Neoantigens and microenvironment in type 1 diabetes: lessons from antitumor immunity. Trends in Endocrinology \& Metabolism. $2016 ; 27(6): 353-62$.

24. S. M. Trigwell, P. M. Radford, S. R. Page, A. C. Loweth, R. F. James, N. G. Morgan, I. Todd. Islet glutamic acid decarboxylase modified by reactive oxygen species is recognized by antibodies from patients with type 1 diabetes mellitus. Clinical \& Experimentat Immunology. 2001; 126(2):242-9.

25. M. van Lummel, G. Duinkerken, P. A. van Veelen, A. de Ru, R. Cordfunke, A. Zaldumbide, I. Gomez-Touriño, S. Arif, M. Peakman, J. W. Drijfhout, B. O. Roep. Posttranslational modification of HLA-DQ binding islet autoantigens in type 1 diabetes. Diabetes. 2014; 63(1):237-47. 
26. J. W. McGinty, I. T. Chow, C. Greenbaum, J. Odegard, W. W. Kwok, E. A. James. Recognition of post-translationally modified glutamic acid decarboxylase 65 epitopes in subjects with type 1 diabetes. Diabetes. 2014; 63(9):3033-40.

27. I. Kharroubi, L. Ladrière, A. K. Cardozo, Z. Dogusan, M. Cnop, D. L. Eizirik. Free fatty acids and cytokines induce pancreatic $\beta$-cell apoptosis by different mechanisms: role of nuclear factor-кB and endoplasmic reticulum stress. Endocrinology. 2004; 145(11):5087-96.

28. D. R. Laybutt, A. M. Preston, M. C. Åkerfeldt, J. G. Kench, A. K. Busch, A. V. Biankin, T. J. Biden. Endoplasmic reticulum stress contributes to beta cell apoptosis in type 2 diabetes. Diabetologia. 2007; 50(4):752-63.

29. S. C. Martinez, K. Tanabe, C. Cras-Méneur, N. A. Abumrad, E. Bernal-Mizrachi, M. A. Permutt. Inhibition of Foxo1 protects pancreatic islet $\beta$-cells against fatty acid and endoplasmic reticulum stress-induced apoptosis. Diabetes. 2008; 57(4):846-59.

30. K. Maedler, G. A. Spinas, D. Dyntar, W. Moritz, N. Kaiser, M. Y. Donath. Distinct effects of saturated and monounsaturated fatty acids on $\beta$-cell turnover and function. Diabetes. 2001; 50(1):69-76.

31. D. A. Cunha, P. Hekerman, L. Ladrière, A. Bazarra-Castro, F. Ortis, M. C. Wakeham, F. Moore, J. Rasschaert, A. K. Cardozo, E. Bellomo, L. Overbergh. Initiation and execution of lipotoxic ER stress in pancreatic $\beta$-cells. Journal of cell science. 2008; 121(14):230818.

32. B. O. Roep, M. J. Kracht, M. van Lummel, A. Zaldumbide. A roadmap of the generation of neoantigens as targets of the immune system in type 1 diabetes. Current Opinion in Immunology. 2016; 43:67-73. 
33. M. S. Horwitz, L. M. Bradley, J. Harbertson, T. Krahl, J. Lee, N. Sarvennick. Diabetes induced by Coxsackie virus: initiation by bystander damage and not molecular mimicry. Nature medicine. 1998; 4(7):781-5.

34. B. T. Kurien, R. H. Scofield. Autoimmunity and oxidatively modified autoantigens. Autoimmunity reviews. 2008; 7(7):567-73.

35. D. L. Kaufnan, M. Clare-Salzler, J. Tian, T. Forsthuber, G. S. Ting, P. Robinson, M. A. Atkinson, E. E. Sercarz, A. J. Tobin, P. V. Lehmann. Spontaneous loss of T-cell tolerance to glutamic acid decarboxylase in murine insulin-dependent diabetes. Nature. $1993 ; 366(6450): 69-72$.

36. S. Hong, M. T. Wilson, I. Serizawa, L. Wu, N. Singh, O. V. Naidenko, T. Miura, T. Haba, D. C. Scherer, J. Wei, M. Kronenberg. The natural killer T-cell ligand $\alpha$ galactosylceramide prevents autoimmune diabetes in non-obese diabetic mice. Nature medicine. 2001; 7(9):1052-6.

37. L. Yu, D. Miao, L. Scrimgeour, K. Johnson, M. Rewers, G. S. Eisenbarth. Distinguishing persistent insulin autoantibodies with differential risk: nonradioactive bivalent proinsulin/insulin autoantibody assay. Diabetes 2012; 61(1):179-186.

38. R. Hilbrands, V. A. Huurman, P. Gillard, J. H. L. Velthuis, M. De Waele, C. Mathieu, L. Kaufman, M. Pipeleers-Marichal, Z. Ling, B. Movahedi, D. Jacobs-TulleneersThevissen, D. Monbaliu, D. Ysebaert, F. K. Gorus, B. O. Roep, D. G. Pipeleers, B. Keymeulen.Differences in baseline lymphocyte counts and autoreactivity are associated with differences in outcome of islet cell transplantation in type 1 diabetic patients. Diabetes 2009; 58:2267-2276.

39. Mayo Medical Laboratories, Mayo Medical Laboratories Interpretive Handbook, Mayo Medical Laboratories, Rochester, USA 2005. 
40. D. Miao, K. M. Guyer, F. Dong, L. Jiang, A. K. Steck, M. Rewers, G. S. Eisenbarth GS, L. Yu. GAD65 autoantibodies detected by electrochemiluminescence assay identify high (1) risk for type 1 diabetes. Diabetes. 2013; 62(12):4174-8.

41. L. Yu, F. Dong, D. Miao, A. R. Fouts, J. M. Wenzlau, A. K. Steck. Proinsulin/insulin autoantibodies measured with electrochemiluminescent assay are the earliest indicator of prediabetic islet autoimmunity. Diabetes care. 2013; 36(8):2266-70.

42. M. Evans, C. Sewter, E. Hill. An encoded particle array tool for multiplex bioassays. Assay Drug Dev. Technol. 2003; 1:199-207.

43. J. P. Nolan, L. A. Sklar. Suspension array technology: evolution of flat array paradigm. Trends in Biotech. 2002; 20:9-12.

44. S. C. Chapin, D. C. Pregibon, P. S. Doyle. High throughput flow alignment of barcoded hydrogel microparticles. Lab Chip. 2009; 9:3100-3109.

45. L. Wang, C. Yang, W. Tan. Dual luminophore doped silica nanoparticles for multiplexed signaling. Nano Lett. 2005; 5:37-43.

46. B. He, S. J. Son, S. B. Lee SB. Suspension array with shape coded silica nanotubes for multiplexed immunoassays. Anal. Chem. 2007; 79: 5257-5263.

47. S. Birtwell. H. Morgan. Microparticle encoding technologies for high-throughput multiplexed suspension assays. Integr. Biol. 2009; 1(5-6):345-362.

48. A. A. Al-Ameen, G. Ghosh. Sensitive quantification of vascular endothelial growth factor (VEGF) using porosity induced hydrogel microspheres. Biosensors and Bioelectronics 2013; 49:105-110.

49. M. A. Al-Ameen, J. Li, D. G. Beer, G. Ghosh. Sensitive, quantitative, and highthroughput detection of angiogenic markers using shape-coded hydrogel microparticles. Analyst. 2015; 140(13):4530-9. 
50. Z. Zhao, M. A. Al-Ameen, K. Duan, G. Ghosh, J. F. Lo. On-chip porous microgel generation for microfluidic enhanced VEGF detection. Biosensors and Bioelectronics. $2015 ; 74: 305-12$.

51. K. Dutan, G. Ghosh, J. F. Lo, On-chip porous microgel based insulin autoantibody (IAA) detection for type I diabetes diagnostics, MicroTAS 2016, 0956.

52. G. Huang, Y. Xiang, L. Pan, X. Li, S. Luo, Z. Zhou. Zinc transporter 8 autoantibody (ZnT8A) could help differentiate latent autoimmune diabetes in adults (LADA) from phenotypic type 2 diabetes mellitus. Diabetes/metabolism research and reviews. 2013; $29(5): 363-8$.

53. F. K. Gorus, E. V. Balti, I. Vermeulen, S. Demeester, A. Van Dalem, O. Costa, H. Dorchy, S. Tenoutasse, T. Mouraux, C. De Block, P. Gillard. Screening for insulinoma antigen 2 and zinc transporter 8 autoantibodies: a cost- effective and age- independent strategy to identify rapid progressors to clinical onset among relatives of type 1 diabetic patients. Clinical \& Experimental Immunology. 2013; 171(1):82-90.

54. M. Fabris, S. Zago, M. Liguori, M. T. Trevisan, M. Zanatta, A. Comici, G. Zanette, E. Carlin,F. Curcio, E. Tonutti. Anti-zinc transporter protein 8 autoantibodies significantly improve the diagnostic approach to type 1 diabetes: an Italian multicentre study on paediatrie patients. Auto-immunity highlights. 2015; 6(1-2):17.

55. J. Kopeček. Hydrogel biomaterials: a smart future?. Biomaterials. 2007; 28(34):5185-92.

56. E. Cosgriff-Hernandez, M. S. Hahn, B. Russell, T. Wilems, D. Munoz-Pinto, M. B. Browning, J. Rivera, M. Höök. Bioactive hydrogels based on designer collagens. Acta biomaterialia. 2010; 6(10):3969-77.

57. M. C. Branco, D. J. Pochan, N. J. Wagner, J. P. Schneider. The effect of protein structure on their controlled release from an injectable peptide hydrogel. Biomaterials. 2010; 31(36):9527-34 
58. S. Frokjaer, D. E. Otzen. Protein drug stability: a formulation challenge. Nature reviews drug discovery. 2005; 4(4):298-306.

59. M. Ye, S. Kim, K. Park. Issues in long-term protein delivery using biodegradable microparticles. Journal of Controlled Release. 2010; 146(2):241-60.

60. R. E. Sallach, W. Cui, F. Balderrama, A. W. Martinez, J. Wen, C. A. Haller, J. V. Taylor, E. R. Wright, R. C. Long, E. L. Chaikof. Long-term biostability of selfassembling protein polymers in the absence of covalent crosslinking. Biomaterials. $2010 ; 31(4): 779-91$.

61. L. Li, Q. Wang, J. Feng, L. Tong, B. Tang. Highly sensitive and homogeneous detection of membrane protein on a single living cell by aptamer and nicking enzyme assisted signa amplification based on microfluidic droplets. Analytical chemistry. 2014; $86(10): 5101-7$

62. H. Zhang, G. Jenkins, Y. Zou, Z. Zhu, C. J. Yang. Massively parallel single-molecule and single-cell emulsion reverse transcription polymerase chain reaction using agarose droplet microfluidics. Analytical chemistry. 2012; 84(8):3599-606.

63. E. Brouzes, M. Medkova, N. Savenelli, D. Marran, M. Twardowski, J. B. Hutchison, J. M. Rothberg, D. R. Link, N. Perrimon, M. L. Samuels. Droplet microfluidic technology for single-cell high-throughput screening. Proceedings of the National Academy of Sciences. 2009; 106(34):14195-200.

64. A. Huebner, M. Srisa-Art, D. Holt, C. Abell, F. Hollfelder, J. B. Edel. Quantitative detection of protein expression in single cells using droplet microfluidics. Chemical Communications. 2007; (12):1218-20.

65. D. Chen, W. Du, Y. Liu, W. Liu, A. Kuznetsov, F. E. Mendez, L. H. Philipson, R. F. Ismagilov. The chemistrode: a droplet-based microfluidic device for stimulation and 
recording with high temporal, spatial, and chemical resolution. Proceedings of the National Academy of Sciences. 2008; 105(44):16843-8.

66. C. J. Easley, J. V. Rocheleau, W. S. Head, D. W. Piston. Quantitative measurement of zinc secretion from pancreatic islets with high temporal resolution using droplet-based microfluidics. Analytical chemistry. 2009; 81(21):9086-95.

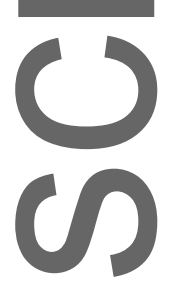

Summary:

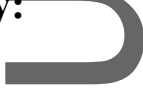

To advance the state of the art suspension assays for diabetes antibodies, porous hydrogel droplets were leveraged in microfluidic serpentine arrays to enhance the detection of antibodies against insulin, GAD, and IA-2. The technique enabled sensitive detections in a single volume, multiplexed format, a breakthrough necessary for the adoption of the assay for clinical screening and monitoring in the future.

\section{TOC Graphics:}
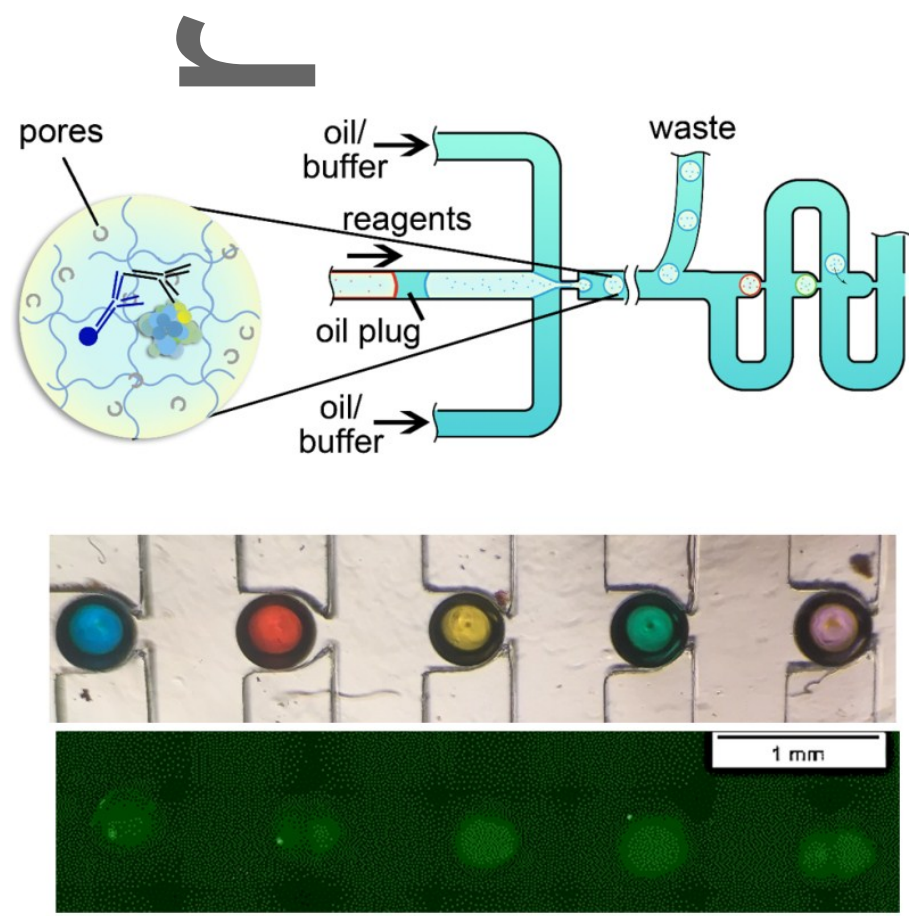

This article is protected by copyright. All rights reserved. 


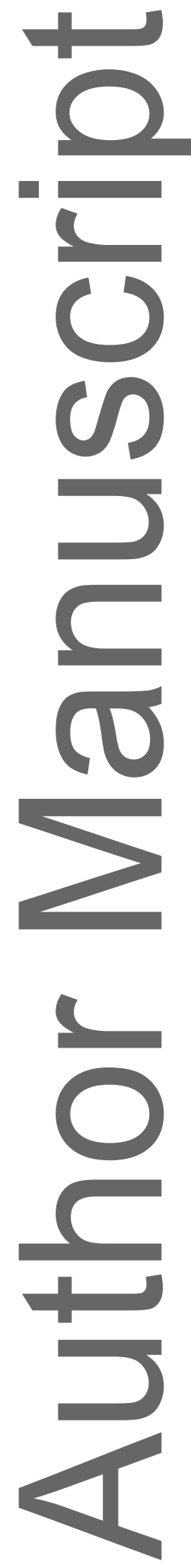

This article is protected by copyright. All rights reserved. 